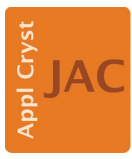

JOURNAL OF

APPLIED

CRYSTALLOGRAPHY

ISSN 1600-5767

Accepted 4 September 2015

Edited by M. Nespolo, Université de Lorraine, France

Keywords: book reviews; X-ray scattering

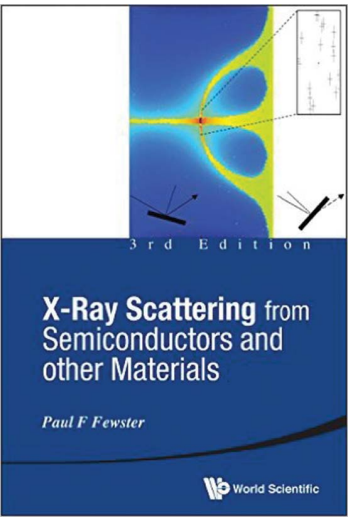

(C) 2015 International Union of Crystallography

\section{X-ray Scattering from Semiconductors and Other Materials. 3rd Ed. By Paul F. Fewster. Singapore: World Scientific Press, 2015. Pp. 493. Price GBP 65.00, USD 99.00. ISBN 9789814436922.}

\author{
Brian Tanner*
}

Department of Physics, Durham University, South Road, Durham DH1 3LE, UK. *Correspondence e-mail: b.k.tanner@durham.ac.uk

X-ray scattering has now become a standard metrology within the semiconductor industry and as such is regularly used by analytical scientists and engineers who are not experts in X-ray physics, but who rely on the validity of the data that are coming from commercial X-ray instruments. For them, this is an ideal book, which distils the knowledge accrued by Paul Fewster over a long and distinguished career into a compact account of how the techniques may be used to understand the structure of advanced materials. Fewster, as one of the best experimentalists of his generation, gives insights into the subtleties of data extraction, instrument alignment and choice of configuration to obtain optimal performance from a system.

Like the first two editions, the book begins with an introduction to semiconducting materials and the influence of distortions on their structure, emphasizing the importance of epitaxial relationships in modern device materials. The second chapter contains the theory of X-ray scattering. This, as with the fourth chapter on instrument selection, is a mine of information and it provides a detailed exposition of the physics needed to understand how materials properties can be deduced from the collected data. These two chapters are not 'novice friendly'; the mixing of quantum and classical ideas is not helpful and the reader is taken to quite recent research work before the basics will have been grasped. Use of text boxes to differentiate complex and second-order ideas from the main exposition would have been helpful. The great value of these chapters is for the diffractometer user who, having thought that understanding of Bragg 's law and thickness fringes was all that was necessary, encounters apparent anomalies in data that need a deeper understanding. For such analytical scientists this book is an essential tool. The third chapter describes the properties of X-ray optical components used for scattering measurements and shows clearly how different devices can be combined to select appropriate instrument functions. The last chapter gives a number of excellent case studies of how materials parameters can be extracted confidently from scattering data. This is set out in sequence of increasing complexity of interpretation and includes valuable information on how to treat imperfect systems, extending from single crystals to polycrystalline materials.

It is a pity that the criticisms of Ullrich Pietsch (2005) relating to the second edition have not been heeded. The presentation is poor in several respects. Fonts of equations vary wildly and unexpectedly. Graph axes are still missing units; diagrams, equations and tables still appear without definition of symbols. The book is deliberately focused at laboratory instrument users and the huge advances made at synchrotron radiation sources get barely a mention. Nevertheless for the specific audience, this new edition is a most valuable resource. The extra material included does make the purchase of another edition worthwhile.

\section{References}

Pietsch, U. (2005). J. Appl. Cryst. 38, 239. 